\title{
Magnetic Resonance Imaging Diagnosis of Volvulus through Mesenteric Defect in Neonate
}

\author{
Scott Leopold, MD ${ }^{1} \quad$ Mohammed Al-Qaraghouli, MD ${ }^{2} \quad$ Naveed Hussain, MD ${ }^{3} \quad$ Christine Finck, MD \\ ${ }^{1}$ Division of Emergency Medicine, Department of Pediatrics, \\ Nationwide Children's Hospital, Columbus, Ohio \\ 2 Division of Maternal-Fetal Medicine, Department of Obstetrics and \\ Gynecology, University of Connecticut Health Center, Farmington, \\ Connecticut \\ Address for correspondence Naveed Hussain, MBBS, MD, DCH, \\ Connecticut Children's Medical Center, University of Connecticut \\ School of Medicine, 263 Farmington Avenue, Farmington, \\ CT 06030-2948 (e-mail: Hussain@uchc.edu).
}

${ }^{3}$ Division of Neonatology, Department of Pediatrics, Connecticut Children's Medical Center, Hartford, Connecticut

${ }^{4}$ Department of Pediatric Surgery, Connecticut Children's Medical Center, Hartford, Connecticut

Am J Perinatol Rep 2016;6:e239-e242.

\begin{abstract}
Keywords

- internal hernia

- midgut volvulus

- MRI

Antenatal midgut volvulus is a rare surgical emergency in which bowel is severely compromised. Rarely the etiology is a mesenteric defect. Early diagnosis is essential and lifesaving in the immediate newborn period. Typically upper gastrointestinal or ultrasound imaging can be suggestive of the diagnosis of volvulus in the neonate. Sometimes, however, the diagnosis may be elusive. Herein, we report on the use of neonatal magnetic resonance imaging to diagnose a midgut volvulus that occurred through a congenital mesenteric defect.
\end{abstract}

Congenital volvulus is a life-threatening condition, both for the fetus and for the newborn. A volvulus is a twist of small bowel loops or a proximal part of the colon around the mesenteric artery or its branches. Midgut volvulus most commonly presents during the first year of life, especially in the first month (60\% of the cases). ${ }^{1}$ When it occurs antenatally, it is almost always secondary to malrotation of the fetal intestines during embryogenesis or congenital anomalies such as omphalocele, gastroschisis, intestinal atresia, and annular pancreas. ${ }^{1,2}$ On the other hand, the etiology of volvulus without malrotation is unknown and associated anomalies are rare. ${ }^{1,2}$ Very rarely does antenatal midgut volvulus occur due to a mesenteric defect. ${ }^{3}$ The potential consequence of volvulus is severe necrosis of the intestinal wall. More than half of the reported cases with fetal midgut volvulus either died in utero or were aborted. ${ }^{4}$ Prenatal diagnosis of midgut volvulus is difficult. Additionally, identification of a volvulus is routinely made with contrast studies. We report on the use of neonatal magnetic resonance imaging (MRI) to diagnose a midgut volvulus that occurred through a congenital mesenteric defect. The pathognomonic features

received

April 20, 2016

accepted

May 16, 2016
DOI http://dx.doi.org/ 10.1055/s-0036-1584548. ISSN 2157-6998. indicative of fetal volvulus on postnatal ultrasound (US) and MRI are discussed.

\section{Case Report}

A 35-year-old gravida 6, para 3 presented to her obstetrician at 32 weeks with the history of decreased fetal movements over a 1-day period. Nonstress test (NST) was nonreactive with fetal tachycardia initially, which had resolved to baseline of 150 s, but biophysical profile (BPP) score was 6/10. The patient was advised to return the following day for reevaluation. NST was again nonreactive with episodes of tachycardia into the 170s and BPP score was 6/10. Decision was made by the facility to transfer the patient to a tertiary maternal-fetal medicine center for further evaluation and treatment. She had declined genetic screening this pregnancy and reported that her 18 weeks anomaly scan was normal. Medical and surgical history was relevant for three cesarean sections. Physical examination was normal except for mild bilateral pedal edema. On evaluation at the tertiary center the fetal heart rate baseline was in the 160 s with minimal variability.
Copyright $\odot 2016$ by Thieme Medical Publishers, Inc., 333 Seventh Avenue, New York, NY 10001, USA. Tel: +1(212) 584-4662.
License terms

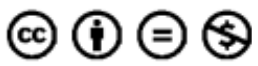


No accelerations and occasional variable decelerations to a fetal heart rate of 150 were noted. The uterus was not contracting. BPP was performed and revealed a score of $2 /$ 10 (for adequate amniotic fluid index (AFI) only). Due to a nonreassuring fetal test and declining biophysical profile scores an emergent cesarean section was performed to deliver a female infant with birth weight 2,045 $\mathrm{g}$ and Apgar scores of 5 and 8 at 1 and 5 minutes, respectively. The infant was immediately transferred to the neonatal intensive care unit where she was subsequently intubated for progressive respiratory distress and was noted on abdominal examination to have a firm 5 to $6 \mathrm{~cm}$ wide and $3 \mathrm{~cm}$ long midline abdominal mass with surrounding blanching of the skin around the umbilicus. She did not have any bilious gastric output. Her vital signs were stable after intubation but she had a significant acidosis with a pH of 7.18 and mild anemia (hematocrit: 35.4\%). Serial plain abdominal radiographs showed gas initially in the stomach and later gas had advanced from the stomach into the adjacent loops of small bowel but no gas was identified in the right abdomen or in lower bowel (-Fig. 1). An abdominal US study was done emergently and showed a sausage-shaped fluid filled mass $5.3 \times 2.8 \times 1.2 \mathrm{~cm}$ in the right upper quadrant. Because of the clinical suspicion of intestinal obstruction the infant was transferred to a regional neonatal surgery center for evaluation. There, another US was done and showed circumferential loops of dilated bowel filled with fluid and succus entericus.

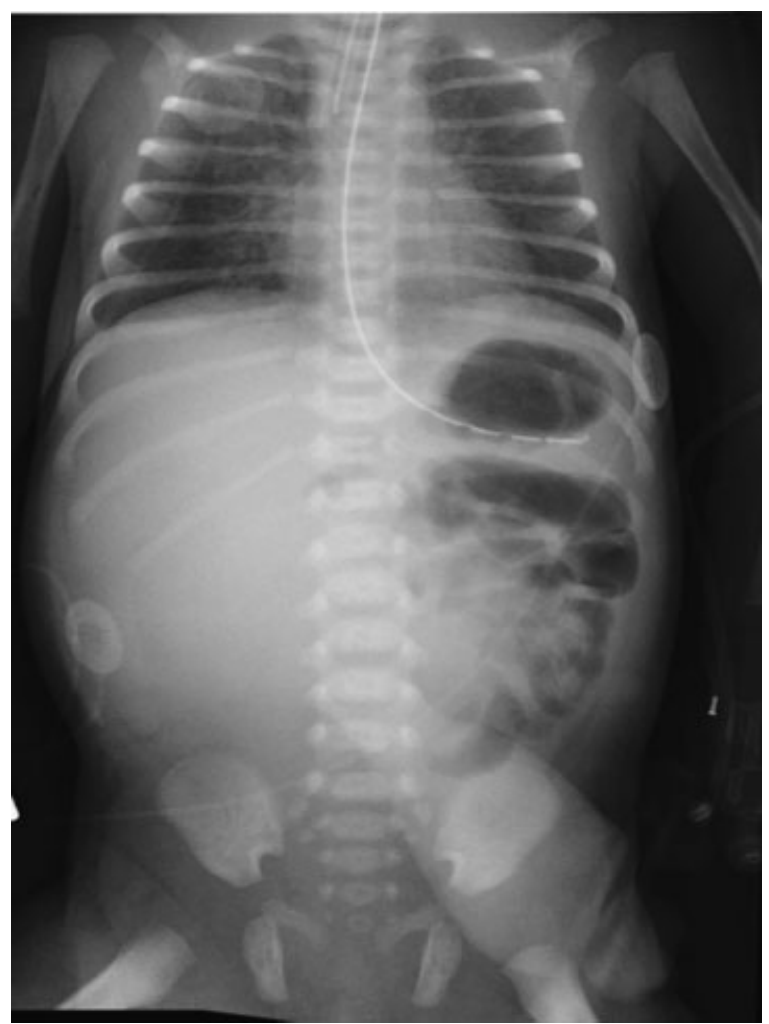

Fig. 1 Plain radiograph of the abdomen in a child with abdominal mass showing air only in the stomach and adjacent small intestine with absence of air in the right abdomen and lower intestine. NG tube is noted in place. NG, nasogastric.
There was a mass located to the right of midline in the right flank region. The exact etiology of this mass was indeterminate. Therefore, further workup to determine the origin and character of the abdominal mass was suggested. An emergent MRI was chosen as it was thought to be able to give the clearest picture of the mass with the least amount of radiation. The MRI demonstrated that the central small bowel was significantly dilated up to $2.6 \mathrm{~cm}$ in diameter (for reference, normal small bowel in this patient measures $\sim 8 \mathrm{~mm}$ in diameter). This abnormally dilated bowel was centrally located slightly to the right and demonstrated a swirling pattern on the coronal views ("whirlpool sign," - Fig. 2). There was concern for closed loop obstruction in the setting of internal hernia. The duodenojejunal junction at ligament of Treitz was in the normal expected position with no evidence of malrotation. Proximal small bowel was air-filled and normal in caliber. There was free fluid in the abdomen (-Fig. 2). The patient was immediately taken to the operating room where an exploratory laparotomy revealed a small Treves' field transmesenteric defect through which approximately $15 \mathrm{~cm}$ of small bowel had herniated and volvulized. The volvulus began from the distal jejunum and proximal ilium. The strangulated necrotic segments were resected and an ileostomy was performed. In retrospect, review of the previously obtained US was suspicious for internal volvulus given the coffee bean sign (-Fig. 3). Subsequently, after surgical repair the patient did well; and at 6 weeks of age had a

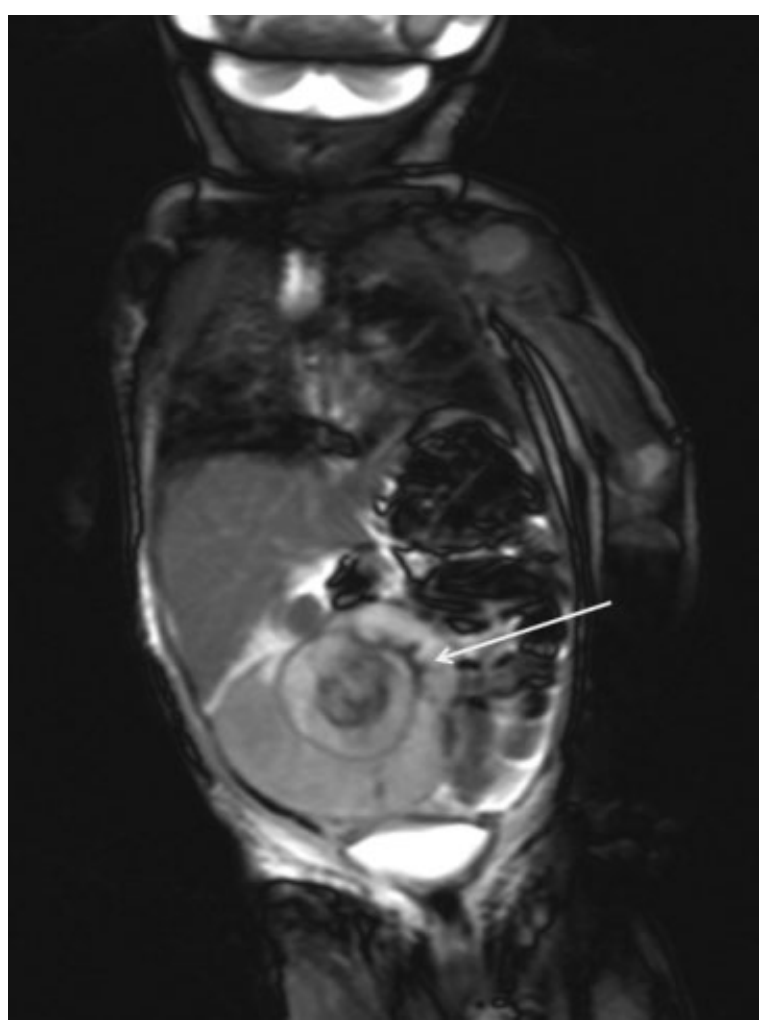

Fig. 2 Chest and abdomen MRI showing dilated central small bowel and swirling pattern of these dilated loops-"whirlpool sign" (arrow). MRI, magnetic resonance imaging. 


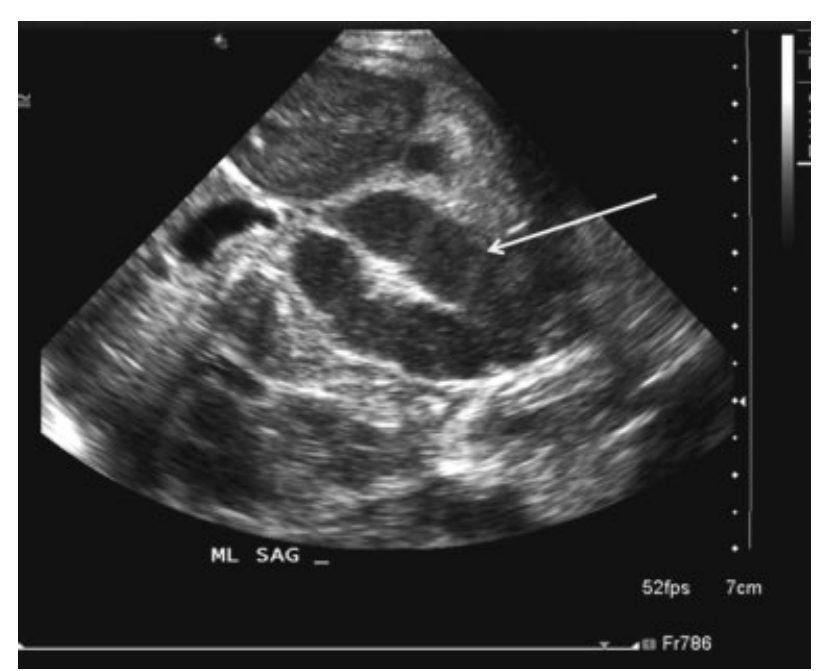

Fig. 3 Abdominal ultrasound that on retrospective review was found to show the "coffee bean sign" (arrow).

takedown of her ileostomy performed along with reanastomosis of the small intestine.

\section{Discussion}

Intrauterine intestinal volvulus is a potentially life-threatening fetal/neonatal condition. Midgut volvulus occurring prenatally is quite rare, and few cases of surviving infants have been reported in the literature. One suggested cause of this condition is internal herniation of the gut through a primary intestinal mesenteric defect (segmental or basilar). ${ }^{1}$ An internal hernia is defined as the herniation of viscera through an anatomic or pathologic opening within the confines of the peritoneal cavity. Most of the cases are discovered during surgery or autopsy. ${ }^{5}$ The Treves' field congenital hernias, defined as a protrusion of a viscus segment into a mesenteric defect or pouch in Treves' field, are varieties of congenital internal hernia. ${ }^{5}$ Mesenteric defects, especially Treves field pouch hernias, are especially dangerous since without a limiting hernia sac, large segments of bowel can become strangulated. ${ }^{5}$ Midgut volvulus is diagnosed antenatally in some cases. US is the imaging method of choice for screening and evaluating fetal anomalies, particularly when an abdominal mass is present. Fluoroscopic studies are indicated for evaluation of malrotation and can be helpful in midgut volvulus depending on how proximal the volvulus is. MRI, on the other hand, can provide excellent discrimination among soft tissues which makes it a useful complementary noninvasive technique in the study of morphology and pathology. To date, no studies, have demonstrated any definite risk to the neonate when MR scanners are operated within the regulatory guidelines set forth by the U.S. Food and Drug Administration and other regulatory agencies. ${ }^{6}$ The drawbacks of using an MRI are the cost, availability, and time required to complete the imaging. In our case, the neonatal presentation of a mass with inconclusive US results led to an emergent MRI. It is highly unusual to diagnose a midgut volvulus in a neonate in this fashion; however, the
MRI gave excellent visualization. Volvulus should be suspected antenatally when polyhydramnios, intestinal dilatation, ascites, and/or signs of fetal anemia can be seen on US assessment. ${ }^{7-9}$ In our case, the AFI was within normal limits but the other four parameters of BPP were all abnormal, suggesting that the fetus was in distress and this normal AFI was a false-negative finding because of the volvulus. Jéquier et al reported a case of volvulus without malrotation in a twin fetus and they concluded that serial US examinations of the bowel are useful for the diagnosis of antenatal small-bowel volvulus. ${ }^{10}$ Steffensen et al reported another case of an antenatal midgut volvulus that was demonstrated on abnormal antenatal US without the pathognomonic whirlpool sign being found prenatally, the definite diagnosis was only made during autopsy. ${ }^{11}$ In a similar manner, Has et al suggested that definitive prenatal diagnosis of midgut volvulus is possible by visualizing twisted bowel loops even if the whirlpool sign is absent on Doppler examination. ${ }^{12}$ In our patient, rapidly dropping BPP scores necessitated an emergent cesarean section and a detailed antenatal US and fetal MRI could not be attained. Given the clinical picture, persistence in evaluation led to successful diagnosis and treatment.

Most cases of midgut volvulus only show nonspecific findings of small bowel obstruction on US. ${ }^{13}$ There have been reports of US diagnosis of prenatal non malrotation volvulus by a "coffee bean sign" which describes the appearance of dilated bowel loops. ${ }^{2,12}$ Here, a central linear density formed by the double thickness of the apposed bowel wall bisects the dilated loop representing the cleft of a coffee bean. ${ }^{14}$ This sign has not been previously described in a postnatal US in a newborn with nonmalrotation volvulus.

In the neonatal period, the diagnosis of a malrotation associated midgut volvulus can be easier than prenatally but an upper gastrointestinal (GI) series and computed tomography scan are not always sufficiently sensitive to diagnose an internal hernia. ${ }^{15}$ In one study, the radiographic diagnosis of 23 neonates with bilious vomiting was evaluated. In cases where the US can definitively diagnose a volvulus, its accuracy precludes the need for further diagnostic tests. However, if it is inconclusive, further radiological contrast studies were needed to make the final diagnosis. ${ }^{16}$ In our case, follow-up T2 MRI demonstrated swirling loops of bowel, a classic sign of internal hernia, and a normally localized duodenojejunal junction, discounting the possibility of malrotation. When obstruction is due to malrotation with Ladd band or volvulus, the loops of the small intestine can be seen lying mostly on the right side of the abdomen while the colon is on the left. ${ }^{17}$ MRI has the advantage over US in differentiating the liver and bowel loops from lung tissue or masses and because of the characteristic signal intensity. ${ }^{18}$ Unfortunately, preoperative diagnosis is not always possible, as reported in Kayastha case report where surgery was done based on a preoperative diagnosis of neonatal intestinal obstruction secondary to meconium ileus, but at laparotomy volvulus of distended small bowel was found. ${ }^{19} \mathrm{~A}$ case was reported by Ohuoba et al, in 2013 in which the 24 weeks antenatal US scan was normal but another scan at 36 weeks showed 
striking tubular dilatation of the lower digestive tract raising concern for complete intestinal obstruction (coffee bean sign) without evidence of fetal anemia. Infant was delivered by cesarean section for repetitive late decelerations, and preoperative diagnosis was made by upper GI series with contrast but no MRI was done. ${ }^{20}$ Immediate surgical management of these cases is life saving and delay in diagnosis can be fatal. Preterm delivery and surgical intervention should be considered when the diagnosis is made prenatally. ${ }^{2,12}$ Park et al in their report noted that delays in diagnosis are likely in such cases, as physicians tend to doubt or not suspect the possibility of intrauterine volvulus because it is so rare. ${ }^{2}$ In our case, with no antenatal diagnosis (or even suspicion), the fluid-filled, palpable abdominal mass in a relatively stable newborn did not immediately raise suspicions of a volvulus. However, the use of immediate MRI was able to establish the diagnosis. We recognize the limitations of MRI including cost, time required to conduct the examination, and availability. In our case MRI was critical in establishing a diagnosis preoperatively and the US findings of "coffee bean sign" were only discovered in retrospect. If there is clinical suspicion with equivocal imaging findings, we recommend immediate operative exploration, as failure to do so could be deleterious.

\section{References}

1 Molvarec A, Bábinszki A, Kovács K, Tóth F, Szalay J. Intrauterine intestinal obstruction due to fetal midgut volvulus: a report of two cases. Fetal Diagn Ther 2007;22(1):38-40

2 Park JS, Cha SJ, Kim BG, et al. Intrauterine midgut volvulus without malrotation: diagnosis from the 'coffee bean sign'. World J Gastroenterol 2008;14(9):1456-1458

3 Page MP, Ricca RL, Resnick AS, Puder M, Fishman SJ. Newborn and toddler intestinal obstruction owing to congenital mesenteric defects. J Pediatr Surg 2008;43(4):755-758

4 Crisera CA, Ginsburg HB, Gittes GK. Fetal midgut volvulus presenting at term. J Pediatr Surg 1999;34(8):1280-1281

5 Vaos G, Skondras C. Treves' field congenital hernias in children: an unsuspected rare cause of acute small bowel obstruction. Pediatr Surg Int 2007;23(4):337-342
6 Tocchio S, Kline-Fath B, Kanal E, Schmithorst VJ, Panigrahy A. MRI evaluation and safety in the developing brain. Semin Perinatol 2015;39(2):73-104

7 Nakagawa T, Tachibana D, Kitada K, et al. A case of fetal intestinal volvulus without malrotation causing severe anemia. Jpn Clin Med 2015;6:1-3

8 Kaba M, Oksuzoglu A, Kaba G, Timur H, Akbaba E, Turgut K. Fetal Midgut Volvulus with a Cystic Appearance, Accompanying a Sinus Rhythm and an Increased Peak Systolic Velocity without Anemia. Case Rep Obstet Gynecol 2015;2015:354619

9 Kornacki J, Czarnecka M, Błaszczyński M, et al. Congenital midgut volvulus associated with fetal anemia. Fetal Diagn Ther 2010; 28(2):119-122

10 Jéquier S, Hanquinet S, Bugmann P, Pfizenmaier M. Antenatal small-bowel volvulus without malrotation: ultrasound demonstration and discussion of pathogenesis. Pediatr Radiol 2003; 33(4):263-265

11 Steffensen TS, Gilbert-Barness E, DeStefano KA, Kontopoulos EV. Midgut volvulus causing fetal demise in utero. Fetal Pediatr Pathol 2008;27(4-5):223-231

12 Has R, Gunay S. 'Whirlpool' sign in the prenatal diagnosis of intestinal volvulus. Ultrasound Obstet Gynecol 2002;20(3): 307-308

13 Miyakoshi K, Ishimoto $\mathrm{H}$, Tanigaki S, et al. Prenatal diagnosis of midgut volvulus by sonography and magnetic resonance imaging. Am J Perinatol 2001;18(8):447-450

14 Chakraborty A, Ayoob A, DiSantis D. Coffee bean sign. Abdom Imaging 2015;40(7):2904-2905

15 Merrot T, Anastasescu R, Pankevych T, Chaumoître K, Alessandrini P. Small bowel obstruction caused by congenital mesocolic hernia: case report. J Pediatr Surg 2003;38(9):E11-E12

16 Alehossein M, Abdi S, Pourgholami M, Naseri M, Salamati P. Diagnostic accuracy of ultrasound in determining the cause of bilious vomiting in neonates. Iran J Radiol 2012;9(4): 190-194

17 Martin C, Darnell A, Escofet C, Duran C, Pérez V. Fetal MR in the evaluation of pulmonary and digestive system pathology. Insights Imaging 2012;3(3):277-293

18 Saleem SN. Fetal MRI: An approach to practice: A review. J Adv Res 2014;5(5):507-523

19 Kayastha K, Mirza B, Sheikh A. Volvulus of small bowel in a case of simple meconium ileus. APSP J Case Rep 2011;2(1):7

20 Ohuoba E, Fruhman G, Olutoye O, Zacharias N. Perinatal survival of a fetus with intestinal volvulus and intussusception: a case report and review of the literature. AJP Rep 2013;3(2): 107-112 